\title{
Using Metabolomic Approaches to Explore Chemical Diversity in Rice
}

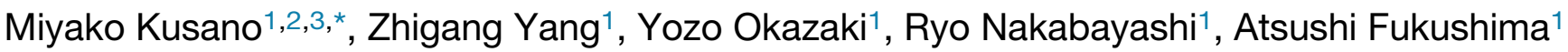 \\ and Kazuki Saito $1,4, *$ \\ ${ }^{1}$ RIKEN Center for Sustainable Resource Science, Yokohama, Kanagawa 230-0045, Japan \\ ${ }^{2}$ Graduate School of Life and Environmental Sciences, University of Tsukuba, Tsukuba, Ibaraki 305-8572, Japan \\ ${ }^{3}$ Precursory Research for Embryonic Science and Technology (PRESTO), Japan Science and Technology Agency (JST), Kawaguchi, Saitama 332-0012, Japan \\ ${ }^{4}$ Graduate School of Pharmaceutical Sciences, Chiba University, Chiba, Chiba 260-8675, Japan \\ *Correspondence: Miyako Kusano (miyako.kusano@riken.jp), Kazuki Saito (kazuki.saito@riken.jp) \\ http://dx.doi.org/10.1016/j.molp.2014.11.010 \\ This is an open access article under the CC BY-NC-ND license (http://creativecommons.org/licenses/by-nc-nd/3.0/).
}

\section{ABSTRACT}

Rice (Oryza sativa) is an excellent resource; it comprises $25 \%$ of the total caloric intake of the world's population, and rice plants yield many types of bioactive compounds. To determine the number of metabolites in rice and their chemical diversity, the metabolite composition of cultivated rice has been investigated with analytical techniques such as mass spectrometry (MS) and/or nuclear magnetic resonance spectroscopy and rice metabolite databases have been constructed. This review summarizes current knowledge on metabolites in rice including sugars, amino and organic acids, aromatic compounds, and phytohormones detected by gas chromatography-MS, liquid chromatography-MS, and capillary electrophoresis-MS. The biological properties and the activities of polar and nonpolar metabolites produced by rice plants are also presented. Challenges in the estimation of the structure(s) of unknown metabolites by metabolomic approaches are introduced and discussed. Lastly, examples are presented of the successful application of metabolite profiling of rice to characterize the gene(s) that are potentially critical for improving its quality by combining metabolite quantitative trait loci analysis and to identify potential metabolite biomarkers that play a critical role when rice is grown under abiotic stress conditions.

Key words: metabolomics, Oryza sativa, chemical diversity, mass spectrometry, gene discovery, QTL analysis

Kusano M., Yang Z., Okazaki Y., Nakabayashi R., Fukushima A., and Saito K. (2015). Using Metabolomic Approaches to Explore Chemical Diversity in Rice. Mol. Plant. 8, 58-67.

\section{INTRODUCTION}

Rice, a member of the Poaceae (Gramineae) family, has a long breeding history. It is one of the major crop grasses particularly in Asian countries because it is a good caloric resource for humans. More than 400000 rice germplasm accessions are stored in gene banks around the world (Toriyama et al., 2005), suggesting that the chemical diversity across rice varieties is large. Natural products chemistry combined with separation science contributes to the investigation of the diversity of chemicals/compounds produced by rice (Figures 1 and 2). Advances in chromatographic techniques, chemometrics, and structural estimation methods make it possible to use metabolomics to explore its chemical diversity.

Metabolomics is one of the major research fields in "omics" science. The analytical targets of metabolomics are biologically synthesized or degraded compounds in organisms. The ultimate goal of plant metabolomics is quantification of the metabolome in plants. It has been estimated that the plant kingdom contains more than 200000 metabolites (Dixon and Strack, 2003). Plants produce essential building blocks such as sugars, amino acids, and phenolics for proteins, starches, and cell wall components that are directly related to agricultural outputs. Various types of bioactive compounds and volatile compounds as secondary metabolites are synthesized to protect plants against biotic and abiotic stress. Their complex metabolism is thought to compensate for their lack of motility. Phytohormones are secondary metabolites that can coordinate cellular activities for germination, growth, protection against stress, and other physiological activities in the life of plants. As crude extracts tend to be used for metabolite profiling in a nontargeted manner, chromatographic separation is usually required (Fukushima and Kusano, 2014), and fractionated compounds

Published by the Molecular Plant Shanghai Editorial Office in association with Cell Press, an imprint of Elsevier Inc., on behalf of CSPB and IPPE, SIBS, CAS. 


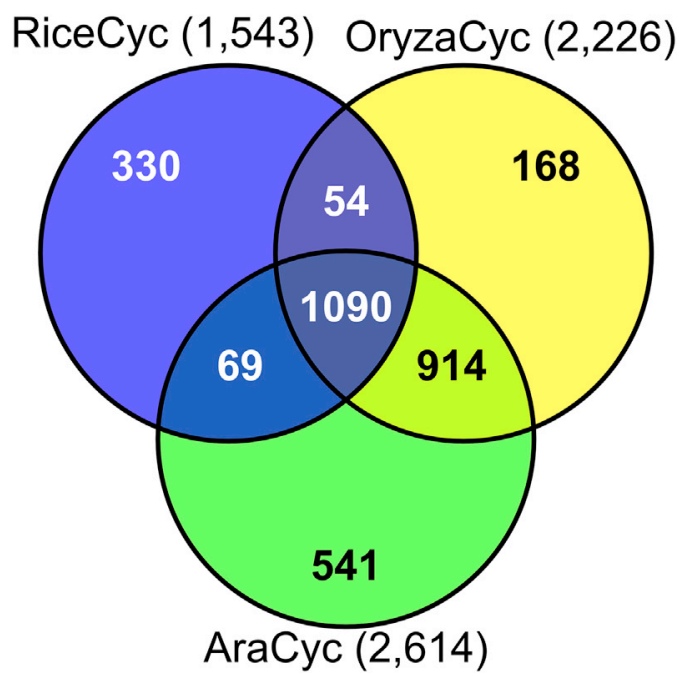

Figure 1. Metabolites Found in RiceCyc and OryzaCyc, but Not in AraCyc.

Based on metabolite IDs (UNIQUE-ID) in RiceCyc (version 3.3, 1543 entries), OryzaCyc (version 1.0, 2226 entries), and AraCyc (version 11.5, 2614 entries), we estimated the proportion of metabolites in rice that was commonly present in the two rice databases. The names of the 54 metabolites in RiceCyc and OryzaCyc are listed in Supplemental Table 1.

are then analyzed by mass spectrometry (MS) and/or nuclear magnetic resonance (NMR) spectroscopy. These analytical methods are most commonly used in not only metabolomics (Kim et al., 2011; Kusano et al., 2011a; Okazaki and Saito, 2012) but also natural products chemistry. MS (Kind et al., 2009; Wishart et al., 2009; Strehmel et al., 2014) and tandem mass spectrometry (MS/MS) spectral libraries (Horai et al., 2010; Sawada et al., 2012; Tautenhahn et al., 2012; Kind et al., 2013) and NMR chemical shift libraries (Steinbeck and Kuhn, 2004; Cui et al., 2008) are available for the provisional identification of known and unknown peaks. The estimation of the chemical structure(s) of unknown metabolites in metabolite profile data continues to present challenges (Nakabayashi and Saito, 2013). Even in the metabolite profiles obtained by gas chromatography (GC) plus MS, approximately half of the peaks remain unidentified because of a lack of reference chemicals. It is impossible or very difficult to estimate the chemical structure(s) of secondary metabolites with only MS-based techniques, in particular for unidentified metabolite peaks. However, ultrahigh-resolution MS (e.g., liquid chromatography [LC]) combined with Fourier transform ion cyclotron resonance-MS increased the possibility of precisely estimating the molecular formula of the peaks of secondary metabolites in the model plant Arabidopsis thaliana (Glaser et al., 2014; Morreel et al., 2014) and in onions (Nakabayashi et al., 2013).

In this review, we estimated the number of metabolites in rice by comparing the metabolite names found in publicly available databases (Figure 1). We present the metabolite composition in the kernels and aerial parts of the cultivated rice. Analysis was performed with GC-MS, LC-MS, and capillary electrophoresis (CE)-MS. We also discuss current knowledge on the secondary metabolites produced by rice and their biological activities (Figure 2) as well as the strategies and challenges for using chemometric approaches to select unidentified peaks as candidates for the further estimation of their chemical structure(s) and to estimate the structure(s) of secondary metabolites by combining high-resolution LC-MS data and algorithms. Lastly, we provide examples of the application of metabolomic approaches to detect metabolites that may be critical for improving the quality of rice by metabolite quantitative trait loci (QTL) analysis and by reverse genetic approaches for the potential enhancement of phosphorus depletion resistance in rice cultivars.

\section{PRIMARY AND SECONDARY METABOLITES IN THE RICE METABOLITE PROFILES}

Plants, including the model plant Arabidopsis and rice, can produce primary metabolites via similar metabolic pathways. In the production of secondary metabolites, the existence of specific pathways in individual plant species must be considered. To estimate the number of metabolites that are thought to exist as specific metabolites in rice plants, we used three plant metabolic databases. According to Seaver et al. (2012), a comparison of individual metabolic databases revealed clear differences in the stored compounds (Seaver et al., 2012). When we compared metabolite IDs used in the RiceCyc (Jaiswal et al., 2006), OryzaCyc, and AraCyc in Plant Metabolic Network (http://www. plantcyc.org/tools/tools_overview.faces on www.plantcyc.org) (Figure 1), we found more than 1000 metabolites (e.g., primary metabolites) in these three databases. On the other hand, there were 54 metabolites in the two metabolic databases of rice. Of these, oryzalexins (Akatsuka et al., 1985; Kato et al., 1993; Kato et al., 1994) and momilactones (Kato-Noguchi and Peters, 2013) are known to be specific metabolites produced in rice (Supplemental Table 1).

As the huge diversity of rice cultivars renders the investigation of the chemical diversity of all rice cultivars unrealistic, genetic core collections have been established to obtain manageable but representative selections of rice cultivars. The size of the genetic core collection deposited in several gene banks is relatively small. Examples are the Rice Germplasm Core Set from the International Rice Research Institute (623 accessions; http://iris.irri. org/germplasm/), the GCore collection (18 collections each of which consists of approximately 130 accessions) ( $L i$ et al., 2004), the EMBRAPA Rice Core Collection (242 accessions) (Borba et al., 2009), and the rice diversity research set (67 accessions) (Kojima et al., 2005). Comprehensive metabolite profiling of kernels in the rice diversity research set, two control cultivars, and the Pokkari cultivar used a combination of oneand two-dimensional GC-time-of-flight (TOF)-MS (Kusano et al., 2007) or four different separation techniques applied in TOF-MS, i.e., GC-TOF-MS, CE-TOF-MS, LC coupled to quadruple orthogonal TOF-MS, and LC coupled to ion trapTOF-MS (Redestig et al., 2011b). Multi-MS-based metabolite profiling covered more than $87 \%$ of the chemical diversity of the metabolites when compared to the metabolites listed in RiceCyc (Figure 2). In 70 rice cultivars, 1652 peaks consisting of 156 distinct metabolites and 1496 unknown analytes comprised the metabolite composition of the rice kernels. Among the 156 distinct metabolites, we provisionally identified 
A

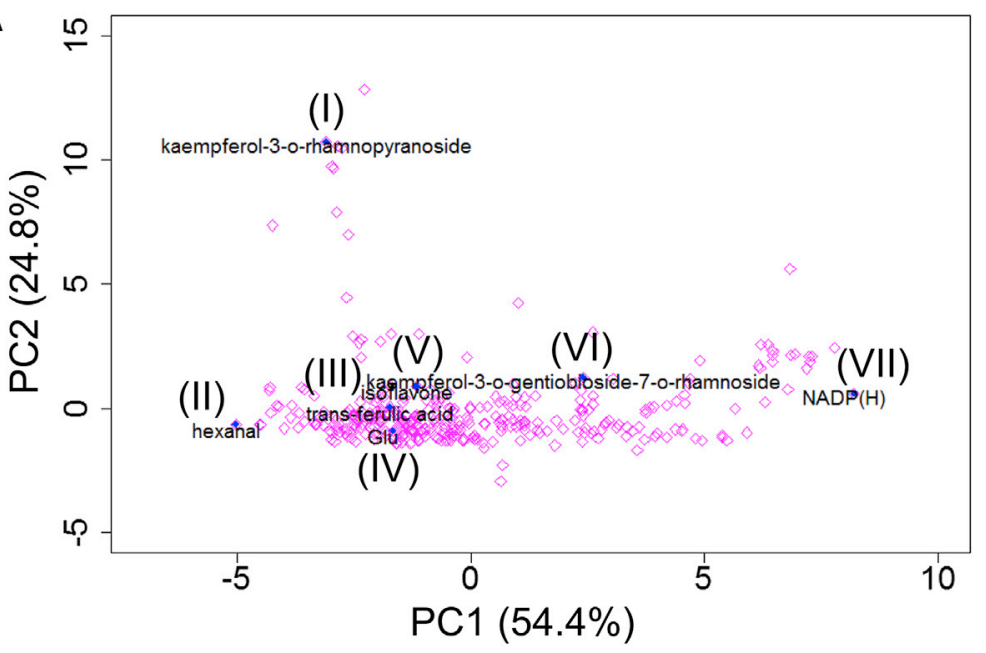

B

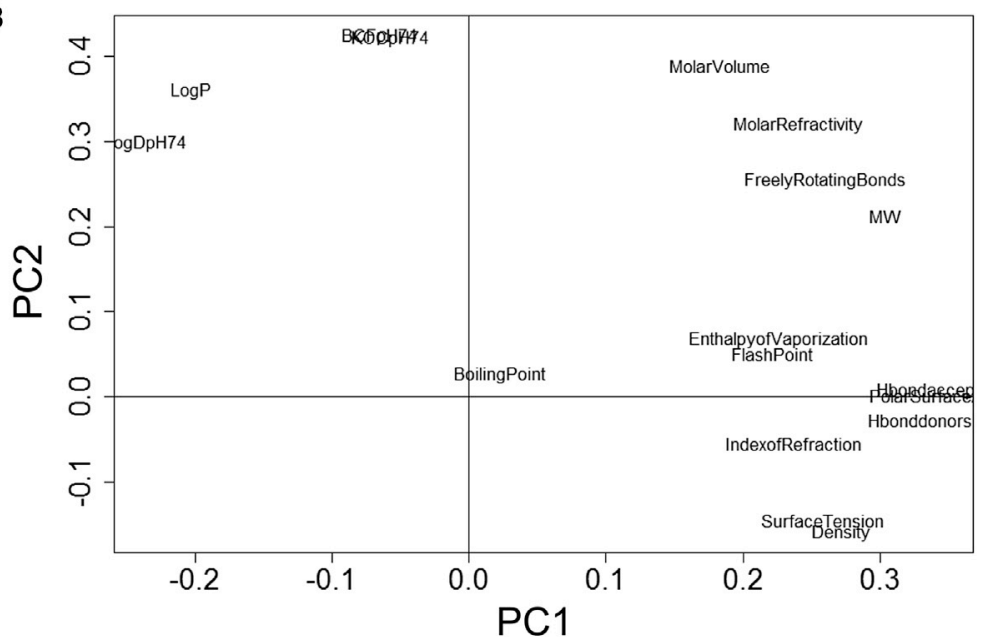

C

(I)<smiles>C[C@@H]1O[C@H](Oc2c(-c3ccc(O)cc3)oc3cc(O)cc(O)c3c2=O)[C@H](O)[C@H](O)[C@H]1O</smiles>

(II)<smiles>CCCCCC=O</smiles>

(III)<smiles>CCOc1cc(/C=C/C(=O)O)ccc1O</smiles><smiles>O=C(O)CCC(O)C(=O)O</smiles>

(V)<smiles>O=c1c(-c2ccccc2)coc2ccccc12</smiles>

$(\mathrm{VI})$

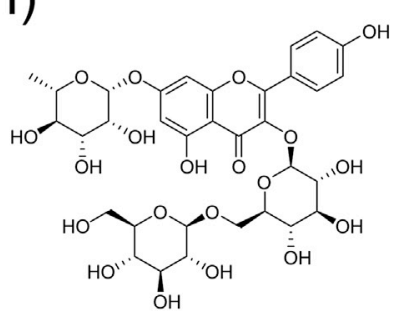

(VII)

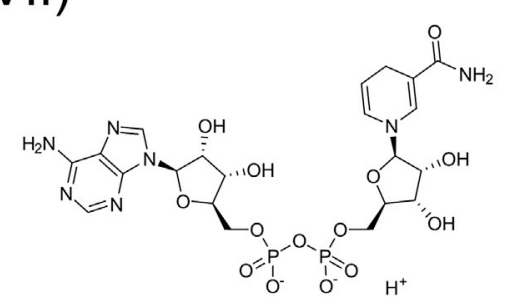

Figure 2. Visualization of the Predicted Chemical Diversity of Metabolites in Rice.

The score scatter (A) and loading plots (B) of principal component analysis (PCA) to estimate the chemical diversity of the 342 metabolites in the RiceCyc database. We used the 17 predicted physicochemical properties of the 342 metabolites in RiceCyc version 3.3 (http://pathway. gramene.org/gramene/ricecyc.shtml) to predict the chemical diversity of rice metabolites by PCA. Of the 1520 compounds in the database, we obtained 342 ChemSpider IDs that could be linked to physiochemical properties, structure information, and chemical identifiers in ChemSpider (http://www.chemspider.com/). The loading plots show that principal component 1 (PC1) tends to be dominated by size- and polarity-related properties. We used the 17 predicted physicochemical properties, i.e., the molecular weight (MW), octanol:water partitioning coefficient (LogP), octanol:water solubility distribution coefficient at $\mathrm{pH} 7.4(\mathrm{LogDpH} 74)$, biological concentration factor at $\mathrm{pH} 7.4$ (BCFpH74), adsorption coefficient at $\mathrm{pH} 7.4$ (KOCpH74), number of $\mathrm{H}$-bond acceptors (Hbondacceptors), number of $\mathrm{H}$-bond donors (Hbonddonors), freely rotating bonds (FreelyRotatingBonds), polar surface area (PolarSurfaceArea), index of refraction (IndexofRefraction), molecular refractivity (MolarRefractivity), molecular volume (MolarVolume), surface tension (SurfaceTension), flash point (FlashPoint), boiling point (BoilingPoint), enthalpy of vaporization (EnthalpyofVaporization), and density (Density). Method details were described by Kusano et al. (2011b). In (C), we list the representative metabolites in the RiceCyc database.

I, kaempferol-3-O-rhamnopyranoside; II, hexanal; III, transferulic acid; IV, glutamate; $\mathrm{V}$, isoflavone; VI, kaempferol-3O-gentiobioside-7-O-rhamnoside; and VII, NADP(H).

levels of indole-3-acetic acid and trans-zeatin, we could detect these phytohormones in the metabolite profile data. Rice kernels contain not only primary metabolites such as sugars, amino and organic acids, fatty acids, and nucleobases but also various types of secondary metabolites. Classic natural products chemistry provides a solid basis for the identification of secondary metabolites. Traitmetabolite association analysis in rice seeds of the original core collection (51 Japonica and 49 Indica accessions) was based on the 121 metabolites detected by LC-tandem MS (MS/MS) and GC-MS (Hu et al., 2014). It captured differences in the metabolite abundance and correlations between Japonica and Indica rice, suggesting that these differences may reflect the extent of metabolic adaptation and the specific phenotypes in Japonica and Indica.

To quantify the metabolite levels in rice leaves, their metabolite composition was investigated with CEMS and a CE-diode array detector (Sato et al., 2004). To visualize the metabolic impact of overexpression of the Arabidopsis NAD kinase

peaks and identified metabolites by comparing their mass spectra and retention times (or indices) with those of the authentic standards. As rice kernels contain relatively higher gene in rice plants, Takahara et al. (2010) used CE-MS and Albinsky et al. (2010) applied GC-MS to characterize the transcription factor gene involved in nitrogen metabolism. By 
<smiles>COc1cc(-c2cc(=O)c3c(O)cc(O)cc3o2)cc(OC)c1O</smiles>

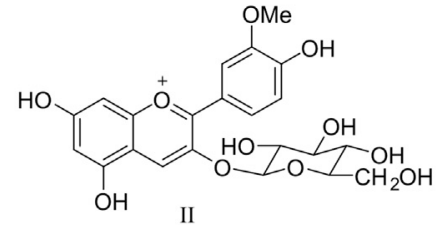<smiles>COc1cc(O)c2c(c1)OC(c1ccc(O)cc1)CC2=O</smiles>

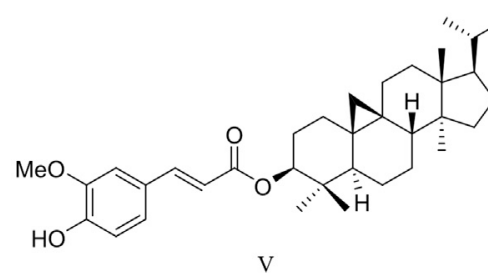

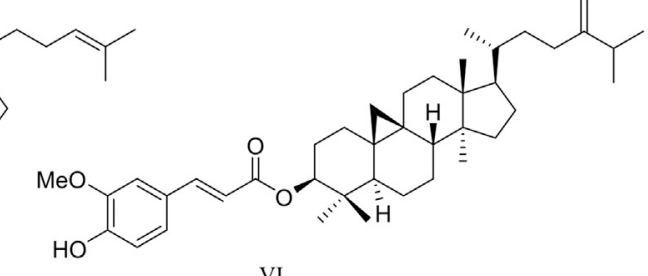

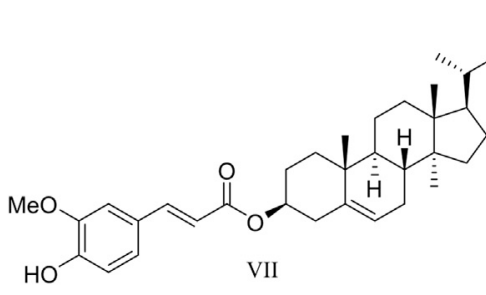
VII<smiles>CCC(CC)C(C)C</smiles>

$\mathrm{V}$<smiles>Oc1ccc(C=CC2CCCCC2)cc1</smiles>

Figure 3. Chemical Structure of Representative Bioactive Metabolites in Rice.

I, cyanindin 3-O-glucoside; II, peonidin 3-Oglucoside; III, tricin; IV, sakuranetin; V, cycloartenol trans-ferulate; $\mathrm{VI}, 24$-methylenecycloartanol transferulate; VII, sitosterol trans-ferulate; and VIII, campesterol trans-ferulate.

These metabolites are introduced in this review. For more details on the bioactive metabolites found in rice, see Supplemental Tables 2 and 3.

sition of aromatic compounds in rice grains. Rice can emit volatile phytohormones such as salicylate, jasmonate (Iwai et al., 2006), and green leaf volatiles (e.g., (Z)-3hexenal) to resist biotic stress (e.g., pathogen infection) (Tong et al., 2012). Ethylene, another volatile phytohormone, is released inside the leaf sheath for a rapid internode elongation response when floating rice is submerged in floods or deep water (Hattori et al., 2009).

For the quantification of gibberellins (Green et al., 1997) and brassinosteroids (Noguchi et al., 1999; Yamamuro et al., 2000), GCMS is used after several fractionations and derivatization. Improvements in the sensitivity of LC-MS have made possible

combining GC-MS and CE-MS techniques, more than 100 metabolites in central metabolism were detected (Redestig et al., 2011b). Classic quantification methods or multiple MSbased platforms were applied to explore metabolite changes in the level of primary metabolites and phytohormones in response to abiotic stresses such as drought (Reguera et al., 2013) and cold or dehydration (Maruyama et al., 2014).

\section{IDENTIFIED VOLATILES (AROMA) AND PHYTOHORMONES IN RICE}

Volatiles and phytohormones are classified as secondary metabolites. More than 300 aroma components have been identified in rice by comparing their mass spectra and those of the authentic standard and/or the mass spectra found in reference libraries (Maga, 1984; Widjaja et al., 1996; Jezussek et al., 2002). Of these, 2-acetyl-1-pyrroline is a major characteristic aroma predominantly detected in scented rice (Buttery et al., 1982; Laguerre et al., 2007; Fitzgerald et al., 2009). The concentration of 2-acetyl-1-pyrroline exhibited a positive correlation with the sensory intensity of aromatic and nonaromatic brown rice cultivars (Itani et al., 2004). Recent developments in headspace collection methods such as solid microphase extraction revealed other flavor components in rice. Zeng et al. (2012) reported a headspace collection of rice bran using solid microphase extraction; the volatiles collected were thermally desorbed by GC-MS (Zeng et al., 2012). They provisionally identified 43 of 76 compounds based on their mass spectra and temperature-programmed retention indices and attempted to quantify the volatile levels with an overall volume integration technique. Such trials can increase our knowledge of the compo- the absolute quantification of 43 molecular species in the four classes of phytohormones: cytokinins, auxins, gibberellins, and abscisic acids (Kojima et al., 2009). Ding et al. (2013) reported a pretreatment method to quantify brassinosteroids without derivatization using LC-MS (Ding et al., 2013). Strigolactones, a novel class of phytohormones, can be detected by LC-MS (Umehara et al., 2008). More than 100 strigolactone derivatives are thought to exist in the plant kingdom (Akiyama and Hayashi, 2006). Of these, a major form of strigolactones in rice is 2'-epi-5-deoxystrigol (Xie et al., 2013; Cardoso et al., 2014); it can be released as a major strigolactone from rice roots, especially when the plants are grown under phosphorusdeficient conditions (Umehara et al., 2010).

\section{BIOACTIVE SECONDARY METABOLITES PRODUCED BY RICE}

Rice produces various types of secondary metabolites (Figure 3). For example, bran, the residue after polishing rice, is brownblack, red, or purple. The pigments are secondary metabolites. Anthocyanins, a subclass of water-soluble flavonoids, are reddish to purple in color and are thought to be major bioactive constituents (e.g., dietary antioxidants) in such colored rice. The primary form of the anthocyanins produced by pigmented rice is cyanidin 3-O-glucoside (I); the secondary form is peonidin 3-O-glucoside (II) (Figure 3). The total anthocyanin content in black rice cultivars is much higher than that in red rice cultivars (Terahara et al., 1994; Abdel-Aal et al., 2006; Yawadio et al., 2007; Hiemori et al., 2009; Yao et al., 2009; Kim et al., 2010; Sompong et al., 2011). Other types of anthocyanins were found in colored rice cultivars (Supplemental Table 2) (Terahara et al., 
1994; Abdel-Aal et al., 2006; Yao et al., 2009), and cyanidin and malvidin were isolated from dark purple rice (Hyun and Chung, 2004). Pigmented rice cultivars contain a higher amount of phenolics, hydroxycinnamate sucrose esters (Tian et al., 2004), and flavonols such as quercetin, kaempferol, and apigenin (Kim et al., 2010; Sriseadka et al., 2012) than nonpigmented rice. Tricin (III in Figure 3) is one of the flavones produced in the leaves and stems of Gramineae and other plants (Zhou and Ibrahim, 2010). Tricin shows antioxidant activity because of its flavone moiety. Quinolone alkaloids were reported in the aleurone layer of anthocyanin-pigmented rice (Chung and Woo, 2001; Chung and Shin, 2007).

Rice bran oil is a uniquely rich source of commercial and nutritional phytochemicals, such as carotenoids and $\gamma$-oryzanol. Carotenoids are yellow-orange pigments mainly found in fruits and vegetables; $\beta$-carotene (provitamin A), lutein, and zeaxanthin have been reported in rice (Parkhi et al., 2005; Lamberts and Delcour, 2008; Kim et al., 2010). $\gamma$-Oryzanol is a specific component in rice bran; it is a mixture of ferulic acid esters with sterols and triterpene alcohols. The major compounds of $\gamma$-oryzanol are cycloartenol trans-ferulate $(\mathrm{V})$, 24-methylenecycloartanol trans-ferulate (VI), sitosterol trans-ferulate (VII), and campesterol trans-ferulate (VIII) (Figure 3) (Xu and Godber, 1999; Akihisa et al., 2000; Xu et al., 2001; Fang et al., 2003; Yu et al., 2007).

Rice plants accumulate secondary metabolites to protect against biotic and abiotic stresses (Kodama et al., 1992; Lin and Kao, 1999; Zuther et al., 2007; Kusano et al., 2011c; Maruyama et al., 2014). For example, copper treatment of rice leaves produced significant changes in the level of polyamine, which are thought to play important roles in many physiological processes (Lin and Kao, 1999; Gill and Tuteja, 2010). Sakuranetin (IV in Figure 3), one of the phytoalexins, is synthesized biochemically from naringenin in rice, cherry bark, and other plant species. The level of sakuranetin, barely detectable in healthy rice leaves, is rapidly increased under biotic and abiotic stress stimuli including UV treatment and pathogen attack (Shimizu et al., 2012).

\section{CURRENT STRATEGIES AND CHALLENGES FOR USING CHEMOMETRIC APPROACHES TO ESTIMATE THE ABUNDANCE AND STRUCTURE OF UNKNOWN METABOLITE PEAKS}

Rice produces commercially important aromas and bioactive compounds (e.g., antioxidants). The identification of unknown compounds and their biological activities is the first step in the detection of a treasure trove of attractive metabolites in the rice metabolome. Spectroscopic techniques including UV-visible spectroscopy, Fourier transform-infrared spectroscopy, MS, and NMR are used to determine the structure of secondary metabolites. Advances in methods to separate compounds (i.e., chromatography) and molecular ions based on their mass-tocharge ratio (i.e., MS) yield high-quality digital data sets. Chemometrics and bioinformatics are applied to estimate the abundance
(Redestig et al., 2011a) and structure (Wolf et al., 2010; Tohge et al., 2011; Nakabayashi et al., 2013; Yang et al., 2013; Morreel et al., 2014; Zhou et al., 2014) of unknown metabolite peaks in plants. Chemometrics using an artificial biological gradient between two specimens is a powerful approach for the generation of semiquantitative calibration curves for all compounds detected including unknown peaks (Redestig et al., 2011a). This method does not require authentic standards and makes it possible to determine whether the calibration curves fit "ideally" as do clear monotonous and asymptotic curves.

There are three strategies to identify/estimate the chemical structure of unknown peaks. They are the application of (i) chromatographic and spectroscopic techniques for the isolation and the elucidation of their structure (Yang et al., 2014), (ii) chemical isotope labeling methods (Nakabayashi et al., 2013; Glaser et al., 2014; Zhou et al., 2014), and (iii) in silico analysis of MS and MS/MS data by computational MS (Wolf et al., 2010; Morreel et al., 2014). The latter is particularly attractive because the separation of all standard compounds, even without labeling, for the identification of unknown metabolites is not possible with strategies (i) and (ii). MetFrag (Wolf et al., 2010) provides a candidate list based on exact mass information stored in the three databases, i.e., KEGG Compound (http://www.genome.jp/kegg/compound/), PubChem (https:// pubchem.ncbi.nlm.nih.gov/), and ChemSpider (http://www. chemspider.com/) (with approximately $16021,37^{6}$, and $23^{6}$ entries, respectively). The required information is the exact mass, which can be obtained by using high-resolution MS or MS/MS; the MetFrag algorithm can generate possible fragmentation patterns of the candidate compounds using the bond dissociation approach. Users can compare the fragmentation patterns generated with their own MS/MS spectra.

Recently, Morreel et al. (2014) developed the candidate substrate-product pair network approach, a novel algorithm that called for the high-throughput structural estimation of 145 secondary metabolites such as glucosinolates, flavonoids, benzenoids, lignans, indoles, and apocarotenoids in the metabolite profiles of Arabidopsis obtained by LC-MS. Based on correlations between the peak abundance of a "candidate substrate" and a "candidate product"as well as the difference in the mass-to-charge ratio between them in the profiles, the candidate substrate-product pair algorithm can select possible candidate pairs. This method can be used to elucidate the structure of unknown peaks produced by rice and other organisms by incorporating LC-MS-based profile data into information obtained by MS/MS fragmentation and the retention time.

\section{APPLICATIONS OF RICE METABOLOMICS: ANALYSIS OF QTL-CONTROLLING METABOLIC DIVERSITY AND DISCOVERY OF POTENTIAL METABOLITE BIOMARKERS ASSOCIATED WITH MITIGATION OF ENVIRONMENTAL STRESSES}

Various types of secondary metabolites produced by rice have been identified using spectroscopic techniques. Metabolomic 
approaches boost metabolite identification, and some of them can be used to improve the quality and yield of rice. As the cultivation and breeding of rice have a long history in Asia, a diverse collection of rice cultivars with different traits including yield, height, and metabolite abundance is available. The development of a strategy for QTL analysis facilitates the identification of useful QTLs that control metabolite accumulation and helps to elucidate systems for the regulation of metabolic networks in various plant species (Fernie and Schauer, 2009; Riedelsheimer et al., 2012; Wen et al., 2014). For gene characterization in primary metabolism, metabolite QTLs of Arabidopsis (Keurentjes et al., 2008; Lisec et al., 2008; Rowe et al., 2008), tomato (Schauer et al., 2006; Schauer et al., 2008), and potato (Carreno-Quintero et al., 2012) have been investigated. However, few published mQTL analyses have focused on primary metabolites in rice (Ying et al., 2012). Rather, investigators performed trait-metabolite association analysis (Kusano et al., 2012; Degenkolbe et al., 2013; Zhao et al., 2013; Hu et al., 2014). The effective use of metabolomics and QTL analysis using mapping populations to delineate the genetic control of metabolism in rice has been reported. MQTL analysis of the offspring of particular pairs of rice cultivars helped to discover the genetic regions that determine differences in the level of some metabolites between two particular parental lines. For example, with respect to the characterization of genes in secondary metabolism, we reported metabolite QTL analysis of backcrossed inbred lines produced from Japonica and Indica cultivars and identified a key gene regulating specialized metabolism in the flavonoid biosynthetic pathway that evolved in the Indica-type parental line (Matsuda et al., 2012). Also, mQTL analysis of the 210 recombinant inbred lines generated by crossing two elite rice cultivars using 900 metabolite profile data (Gong et al., 2013) yielded the functional characterization of several genes including two flavone malonyltransferases and unknown genes that are thought to be involved in the flavonoid biosynthetic pathway.

Chen et al. (2014) adapted QTL analysis based on genome-wide association study (GWAS) to link single nucleotide polymorphisms and metabolite levels in rice (Chen et al., 2014). They performed metabolite GWAS using the large matrix consisting of 840 metabolite peaks and 524 accessions and extracted 36 genes as potential candidates involved in metabolite changes of physiological and nutritional importance. They successfully characterized or annotated five genes encoding a methyltransferase, a putrescine/agmatine hydroxycinnamoyl acyltransferase, a putative flavone 5 -O-glucosyltranferase, an agmatine hydroxycinnamoyl acyltransferase, and a putative transferase for the production of identified and unknown metabolites. Matsuda et al. (2014) carried out GWAS using the LC-MS-based profile data of 175 Japanese rice accessions leading to identification of 323 associations among 143 single nucleotide polymorphisms and 89 metabolites (Matsuda et al., 2014). Metabolite identification was based on standard compounds, which were isolated and characterized by solid spectroscopic analyses (Yang et al., 2014). Due to the rapid accumulation of whole-genome sequence information on rice (Li et al., 2014), additional trials to associate metabolic diversity with natural variations in various traits in rice populations are under way (Calingacion et al., 2012; Verhoeven et al., 2012; Hu et al., 2014). The findings will help to improve crop designs that address regional preferences in traits including taste and flavor (Fitzgerald et al., 2009; Calingacion et al., 2014).

When a metabolite or a set of metabolites is differentially accumulated in two subspecies of rice, this may be used as a biomarker. Metabolomics has contributed to the identification of metabolite biomarkers that are associated with tolerance of rice to stress including abiotic stress (Degenkolbe et al., 2013; Maruyama et al., 2014) and nutrition starvation (Masumoto et al., 2010; Okazaki et al., 2013), suggesting metabolomic versatility in rice metabolic studies. According to Vance et al. (2003), crop yields on $30-40 \%$ of the world's arable land are reduced because phosphorus is unavailable (Vance et al., 2003). To cope with phosphorus starvation, plants developed various adaptations; lipid remodeling is one of the most intensely studied mechanisms (Härtel et al., 2000; Benning and Ohta, 2005). A typical metabolite change associated with lipid remodeling during phosphorus deprivation is the replacement of membrane phospholipids with nonphosphorus glycerolipids such as sulfoquinovosyl diacylglycerol and digalactosyl diacylglycerol, which promote the remobilization of phosphorus. Our lipidomic profiling of rice grown under phosphorus-controlled conditions showed that a new type of plant lipid, i.e., glucuronosyl diacylglycerol, was inducibly accumulated under phosphorus-limited conditions in rice and Arabidopsis, suggesting a shared physiological significance of this novel lipid under conditions of phosphorus depletion in plants (Okazaki et al., 2013).

\section{CONCLUSIONS AND FUTURE PERSPECTIVES}

We discussed various types of metabolites in rice, including primary metabolites, volatiles, phytohormones, lipids, and other secondary metabolites such as phenolics, flavonols, carotenoids, and alkaloids. Estimation of the chemical diversity in rice by metabolomic approaches is important for the development of new algorithms for the further elucidation of the chemical structure of unknown peaks. Although phytohormones are classified as secondary metabolites, their production is highly complex and strictly regulated at the transcriptional, translational, and metabolic levels. Such sophisticated regulations triggered by a metabolite were uncovered in Arabidopsis, i.e., root elongation via glucose-target-of-rapamycin signaling (Xiong et al., 2013) and trehalose-6-phosphate signaling for flowering (Wahl et al., 2013). Investigation of such mechanisms using "omics" approaches may give rise to the generation of new hypotheses.

Gain-of-function genetic mutations can add new and useful characteristics to improve the quality of rice. For example, "golden rice," generated with a genetic engineering approach, can accumulate $\beta$-carotene in the endosperm (Ye et al., 2000; Paine et al., 2005). The consumption of golden rice by Asian, African, and Latin American children may help to address their vitamin A deficiency. However, field experiments are needed to determine the effects of genetically modified crops grown under various environmental conditions on metabolite composition (e.g., Kusano et al., 2014).

Many investigations have focused on the metabolites of pigmented rice, rice bran, and bran oil because they 
have health-promoting properties such as antioxidant, antiinflammatory, and antihypercholesterolemic activities. The medicinal activities of unique diterpenoid phytoalexins and alkaloids and oryzamutaic acids in rice must be investigated for their health-promoting functions. Estimation of the structural diversity of secondary metabolites in rice is important for phytochemical genomics because genetic and metabolomic approaches have already helped to improve the nutritional quality of other crops (Fernie and Schauer, 2009).

\section{SUPPLEMENTAL INFORMATION}

Supplemental Information is available at Molecular Plant Online.

\section{FUNDING}

This work was supported in part by the Japan Advanced Plant Science Network and the Strategic International Collaborative Research Program (SICORP) from JST.

\section{ACKNOWLEDGMENTS}

We thank U. Petralia for editorial review. No conflict of interest declared.

Received: July 29, 2014

Accepted: October 16, 2014

Published: November 3, 2014

\section{REFERENCES}

Abdel-Aal, E.S.M., Young, J.C., and Rabalski, I. (2006). Anthocyanin composition in black, blue, pink, purple, and red cereal grains. J. Agric. Food Chem. 54:4696-4704.

Akatsuka, T., Kodama, O., Sekido, H., Kono, Y., and Takeuchi, S. (1985). Novel phytoalexins (oryzalexins A, B and C) isolated from rice blast leaves infected with Pyricularia oryzae .1. Isolation, characterization and biological activities of oryzalexins. Agric. Biol. Chem. Tokyo 49:1689-1694.

Akihisa, T., et al. (2000). Triterpene alcohol and sterol ferulates from rice bran and their anti-inflammatory effects. J. Agric. Food Chem. 48:2313-2319.

Akiyama, K., and Hayashi, H. (2006). Strigolactones: chemical signals for fungal symbionts and parasitic weeds in plant roots. Ann. Bot. 97:925-931.

Albinsky, D., et al. (2010). Metabolomic screening applied to rice FOX Arabidopsis lines leads to the identification of a gene-changing nitrogen metabolism. Mol. Plant 3:125-142.

Benning, C., and Ohta, H. (2005). Three enzyme systems for galactoglycerolipid biosynthesis are coordinately regulated in plants. J. Biol. Chem. 280:2397-2400.

Borba, T.C.D., Brondani, R.P.V., Rangel, P.H.N., and Brondani, C. (2009). Microsatellite marker-mediated analysis of the EMBRAPA Rice Core Collection genetic diversity. Genetica 137:293-304.

Buttery, R.G., Ling, L.C., and Juliano, B.O. (1982). 2-Acetyl-1pyrroline-an important aroma component of cooked rice. Chem. Ind. 23:958-959.

Calingacion, M., et al. (2014). Diversity of global rice markets and the science required for consumer-targeted rice breeding. PLoS One 9:e85106.

Calingacion, M.N., et al. (2012). A genomics and multi-platform metabolomics approach to identify new traits of rice quality in traditional and improved varieties. Metabolomics 8:771-783.

Cardoso, C., et al. (2014). Natural variation of rice strigolactone biosynthesis is associated with the deletion of two MAX1 orthologs. Proc. Natl. Acad. Sci. U S A 111:2379-2384.

Molecular Plant 8, 58-67, January 2015 @ The Author 2015.
Carreno-Quintero, N., et al. (2012). Untargeted metabolic quantitative trait loci analyses reveal a relationship between primary metabolism and potato tuber quality. Plant Physiol. 158:1306-1318.

Chen, W., et al. (2014). Genome-wide association analyses provide genetic and biochemical insights into natural variation in rice metabolism. Nat. Genet. 46:714-721.

Chung, H.S., and Shin, J.C. (2007). Characterization of antioxidant alkaloids and phenolic acids from anthocyanin-pigmented rice (Oryza sativa cv. Heugjinjubyeo). Food Chem. 104:1670-1677.

Chung, H.S., and Woo, W.S. (2001). A quinolone alkaloid with antioxidant activity from the aleurone layer of anthocyanin-pigmented rice. J. Nat. Prod. 64:1579-1580.

Cui, Q., et al. (2008). Metabolite identification via the Madison Metabolomics Consortium Database. Nat. Biotechnol. 26:162-164.

Degenkolbe, T., Do, P.T., Kopka, J., Zuther, E., Hincha, D.K., and Kohl, K.I. (2013). Identification of drought tolerance markers in a diverse population of rice cultivars by expression and metabolite profiling. PLoS One 8:e63637.

Ding, J., Mao, L.J., Yuan, B.F., and Feng, Y.Q. (2013). A selective pretreatment method for determination of endogenous active brassinosteroids in plant tissues: double layered solid phase extraction combined with boronate affinity polymer monolith microextraction. Plant Methods 9:13.

Dixon, R.A., and Strack, D. (2003). Phytochemistry meets genome analysis, and beyond. Phytochemistry 62:815-816.

Fang, N.B., Yu, S.G., and Badger, T.M. (2003). Characterization of triterpene alcohol and sterol ferulates in rice bran using LC-MS/MS. J. Agric. Food Chem. 51:3260-3267.

Fernie, A.R., and Schauer, N. (2009). Metabolomics-assisted breeding: a viable option for crop improvement? Trends Genet. 25:39-48.

Fitzgerald, M.A., McCouch, S.R., and Hall, R.D. (2009). Not just a grain of rice: the quest for quality. Trends Plant Sci. 14:133-139.

Fukushima, A., and Kusano, M. (2014). A network perspective on nitrogen metabolism from model to crop plants using integrated 'omics' approaches. J. Exp. Bot. 65:5619-5630.

Gill, S.S., and Tuteja, N. (2010). Polyamines and abiotic stress tolerance in plants. Plant Signal. Behav. 5:26-33.

Glaser, K., Kanawati, B., Kubo, T., Schmitt-Kopplin, P., and Grill, E. (2014). Exploring the Arabidopsis sulfur metabolome. Plant J. 77:31-45.

Gong, L., et al. (2013). Genetic analysis of the metabolome exemplified using a rice population. Proc. Natl. Acad. Sci. U S A 110:20320-20325.

Green, L.S., Faergestad, E.M., Poole, A., and Chandler, P.M. (1997). Grain development mutants of barley ([alpha]-amylase production during grain maturation and its relation to endogenous gibberellic acid content). Plant Physiol. 114:203-212.

Härtel, H., Dörmann, P., and Benning, C. (2000). DGD1-independent biosynthesis of extraplastidic galactolipids after phosphate deprivation in Arabidopsis. Proc. Natl. Acad. Sci. U S A 97:10649-10654.

Hattori, Y., et al. (2009). The ethylene response factors SNORKEL1 and SNORKEL2 allow rice to adapt to deep water. Nature 460:1026-1030.

Hiemori, M., Koh, E., and Mitchell, A.E. (2009). Influence of cooking on anthocyanins in black rice (Oryza sativa L. japonica var. SBR). J. Agric. Food Chem. 57:1908-1914.

Horai, H., et al. (2010). MassBank: a public repository for sharing mass spectral data for life sciences. J. Mass Spectrom. 45:703-714.

Hu, C., et al. (2014). Metabolic variation between Japonica and Indica rice cultivars as revealed by non-targeted metabolomics. Sci. Rep. 4:5067.

Hyun, J.W., and Chung, H.S. (2004). Cyanidin and malvidin from Oryza sativa Cv. Heugjinjubyeo mediate cytotoxicity against human 
monocytic leukemia cells by arrest of G(2)/M phase and induction of apoptosis. J. Agric. Food Chem. 52:2213-2217.

Itani, T., Tamaki, M., Hayata, Y., Fushimi, T., and Hashizume, K. (2004). Variation of 2-acetyl-1-pyrroline concentration in aromatic rice grains collected in the same region in Japan and factors affecting its concentration. Plant Prod. Sci. 7:178-183.

Iwai, T., Miyasaka, A., Seo, S., and Ohashi, Y. (2006). Contribution of ethylene biosynthesis for resistance to blast fungus infection in young rice plants. Plant Physiol. 142:1202-1215.

Jaiswal, P., et al. (2006). Gramene: a bird's eye view of cereal genomes. Nucleic Acids Res. 34:D717-D723.

Jezussek, M., Juliano, B.O., and Schieberle, P. (2002). Comparison of key aroma compounds in cooked brown rice varieties based on aroma extract dilution analyses. J. Agric. Food Chem. 50:1101-1105.

Kato, H., Kodama, O., and Akatsuka, T. (1993). Oryzalexin-E, a diterpene phytoalexin from UV-irradiated rice leaves. Phytochemistry 33:79-81.

Kato, H., Kodama, O., and Akatsuka, T. (1994). Oryzalexin-F, a diterpene phytoalexin from UV-irradiated rice leaves. Phytochemistry 36:299-301

Kato-Noguchi, H., and Peters, R.J. (2013). The role of momilactones in rice allelopathy. J. Chem. Ecol. 39:175-185.

Keurentjes, J.J., et al. (2008). Integrative analyses of genetic variation in enzyme activities of primary carbohydrate metabolism reveal distinct modes of regulation in Arabidopsis thaliana. Genome Biol. 9:R129.

Kim, H.K., Choi, Y.H., and Verpoorte, R. (2011). NMR-based plant metabolomics: where do we stand, where do we go? Trends Biotechnol. 29:267-275.

Kim, J.K., et al. (2010). Variation and correlation analysis of flavonoids and carotenoids in Korean pigmented rice (Oryza sativa L.) cultivars. J. Agric. Food Chem. 58:12804-12809.

Kind, T., Liu, K.H., Lee do, Y., DeFelice, B., Meissen, J.K., and Fiehn, O. (2013). LipidBlast in silico tandem mass spectrometry database for lipid identification. Nat. Methods 10:755-758.

Kind, T., et al. (2009). FiehnLib: mass spectral and retention index libraries for metabolomics based on quadrupole and time-of-flight gas chromatography/mass spectrometry. Anal. Chem. 81:10038-10048.

Kodama, O., Miyakawa, J., Akatsuka, T., and Kiyosawa, S. (1992). Sakuranetin, a flavanone phytoalexin from ultraviolet-irradiated rice leaves. Phytochemistry 31:3807-3809.

Kojima, Y., Ebana, K., Fukuoka, S., Nagamine, T., and Kawase, M. (2005). Development of an RFLP-based rice diversity research set of germplasm. Breed. Sci. 55:431-440.

Kojima, M., et al. (2009). Highly sensitive and high-throughput analysis of plant hormones using MS-probe modification and liquid chromatography-tandem mass spectrometry: an application for hormone profiling in Oryza sativa. Plant Cell Physiol. 50:1201-1214.

Kusano, M., Fukushima, A., Redestig, H., and Saito, K. (2011a). Metabolomic approaches toward understanding nitrogen metabolism in plants. J. Exp. Bot. 62:1439-1453.

Kusano, M., et al. (2011b). Covering chemical diversity of geneticallymodified tomatoes using metabolomics for objective substantial equivalence assessment. PLoS One 6:e16989.

Kusano, M., et al. (2011c). Metabolomics data reveal a crucial role of cytosolic glutamine synthetase 1;1 in coordinating metabolic balance in rice. Plant J. 66:456-466.

Kusano, M., et al. (2007). Application of a metabolomic method combining one-dimensional and two-dimensional gas chromatography-time-offlight/mass spectrometry to metabolic phenotyping of natural variants in rice. J. Chromatogr. B 855:71-79.
Kusano, M., et al. (2012). Deciphering starch quality of rice kernels using metabolite profiling and pedigree network analysis. Mol. Plant 5:442-451.

Kusano, M., et al. (2014). Assessing metabolomic and chemical diversity of a soybean lineage representing 35 years of breeding. Metabolomics, Advance Access published August 8, 2014. http://dx.doi.org/10.1007/ s11306-014-0702-6.

Laguerre, M., Mestres, C., Davrieux, F., Ringuet, J., and Boulanger, R. (2007). Rapid discrimination of scented rice by solid-phase microextraction, mass spectrometry, and multivariate analysis used as a mass sensor. J. Agric. Food Chem. 55:1077-1083.

Lamberts, L., and Delcour, J.A. (2008). Carotenoids in raw and parboiled brown and milled rice. J. Agric. Food Chem. 56:11914-11919.

Li, C.T., Shi, C.H., Wu, J.G., Xu, H.M., Zhang, H.Z., and Ren, Y.L. (2004). Methods of developing core collections based on the predicted genotypic value of rice (Oryza sativa L.). Theor. Appl. Genet. 108:1172-1176.

Li, J.Y., Wang, J., and Zeigler, R.S. (2014). The 3,000 rice genomes project: new opportunities and challenges for future rice research. GigaScience 3:8.

Lin, C.C., and Kao, C.H. (1999). Excess copper induces an accumulation of putrescine in rice leaves. Bot. Bull. Acad. Sinica 40:213-218.

Lisec, J., et al. (2008). Identification of metabolic and biomass QTL in Arabidopsis thaliana in a parallel analysis of RIL and IL populations. Plant J. 53:960-972.

Maga, J.A. (1984). Rice product volatiles - a review. J. Agric. Food Chem. 32:964-970.

Maruyama, K., et al. (2014). Integrated analysis of the effects of cold and dehydration on rice metabolites, phytohormones, and gene transcripts. Plant Physiol. 164:1759-1771.

Masumoto, C., et al. (2010). Phosphoenolpyruvate carboxylase intrinsically located in the chloroplast of rice plays a crucial role in ammonium assimilation. Proc. Natl. Acad. Sci. U S A 107:5226-5231.

Matsuda, F., et al. (2012). Dissection of genotype-phenotype associations in rice grains using metabolome quantitative trait loci analysis. Plant J. 70:624-636.

Matsuda, F., et al. (2014). Metabolome-genome-wide association study (mGWAS) dissects genetic architecture for generating natural variation in rice secondary metabolism. Plant J. Advance Access published November 3, 2014. http://dx.doi.org/10.1111/tpj.12681.

Morreel, K., et al. (2014). Systematic structural characterization of metabolites in Arabidopsis via candidate substrate-product pair networks. Plant Cell 26:929-945.

Nakabayashi, R., and Saito, K. (2013). Metabolomics for unknown plant metabolites. Anal. Bioanal. Chem. 405:5005-5011.

Nakabayashi, R., et al. (2013). Combination of liquid chromatographyFourier transform ion cyclotron resonance-mass spectrometry with 13C-labeling for chemical assignment of sulfur-containing metabolites in onion bulbs. Anal. Chem. 85:1310-1315

Noguchi, T., et al. (1999). Brassinosteroid-insensitive dwarf mutants of Arabidopsis accumulate brassinosteroids. Plant Physiol. 121:743-752.

Okazaki, Y., and Saito, K. (2012). Recent advances of metabolomics in plant biotechnology. Plant Biotechnol. Rep. 6:1-15.

Okazaki, Y., et al. (2013). A new class of plant lipid is essential for protection against phosphorus depletion. Nat. Commun. 4:1510.

Paine, J.A., et al. (2005). Improving the nutritional value of Golden Rice through increased pro-vitamin A content. Nat. Biotechnol. 23:482-487.

Parkhi, V., et al. (2005). Molecular characterization of marker-free transgenic lines of Indica rice that accumulate carotenoids in seed endosperm. Mol. Genet. Genomics 274:325-336.

Molecular Plant 8, 58-67, January 2015 @ The Author 2015. 
Redestig, H., Kobayashi, M., Saito, K., and Kusano, M. (2011a). Exploring matrix effects and quantification performance in metabolomics experiments using artificial biological gradients. Anal. Chem. 83:5645-5651.

Redestig, H., et al. (2011b). Exploring molecular backgrounds of quality traits in rice by predictive models based on high-coverage metabolomics. BMC Syst. Biol. 5:176.

Reguera, M., Peleg, Z., Abdel-Tawab, Y.M., Tumimbang, E.B., Delatorre, C.A., and Blumwald, E. (2013). Stress-induced cytokinin synthesis increases drought tolerance through the coordinated regulation of carbon and nitrogen assimilation in rice. Plant Physiol. 163:1609-1622.

Riedelsheimer, C., et al. (2012). Genomic and metabolic prediction of complex heterotic traits in hybrid maize. Nat. Genet. 44:217-220.

Rowe, H.C., Hansen, B.G., Halkier, B.A., and Kliebenstein, D.J. (2008). Biochemical networks and epistasis shape the Arabidopsis thaliana metabolome. Plant Cell 20:1199-1216.

Sato, S., Soga, T., Nishioka, T., and Tomita, M. (2004). Simultaneous determination of the main metabolites in rice leaves using capillary electrophoresis mass spectrometry and capillary electrophoresis diode array detection. Plant J. 40:151-163.

Sawada, Y., et al. (2012). RIKEN tandem mass spectral database (ReSpect) for phytochemicals: a plant-specific MS/MS-based data resource and database. Phytochemistry 82:38-45.

Schauer, N., et al. (2006). Comprehensive metabolic profiling and phenotyping of interspecific introgression lines for tomato improvement. Nat. Biotechnol. 24:447-454.

Schauer, N., et al. (2008). Mode of inheritance of primary metabolic traits in tomato. Plant Cell 20:509-523.

Seaver, S.M., Henry, C.S., and Hanson, A.D. (2012). Frontiers in metabolic reconstruction and modeling of plant genomes. J. Exp. Bot. 63:2247-2258.

Shimizu, T., Lin, F., Hasegawa, M., Nojiri, H., Yamane, H., and Okada, K. (2012). The potential bioproduction of the pharmaceutical agent sakuranetin, a flavonoid phytoalexin in rice. Bioengineered 3:352-357.

Sompong, R., Siebenhandl-Ehn, S., Linsberger-Martin, G., and Berghofer, E. (2011). Physicochemical and antioxidative properties of red and black rice varieties from Thailand, China and Sri Lanka. Food Chem. 124:132-140.

Sriseadka, T., Wongpornchai, S., and Rayanakorn, M. (2012). Quantification of flavonoids in black rice by liquid chromatographynegative electrospray ionization tandem mass spectrometry. J. Agric. Food Chem. 60:11723-11732.

Steinbeck, C., and Kuhn, S. (2004). NMRShiftDB-compound identification and structure elucidation support through a free community-built web database. Phytochemistry 65:2711-2717.

Strehmel, N., Kopka, J., Scheel, D., and Bottcher, C. (2014). Annotating unknown components from GC/El-MS-based metabolite profiling experiments using GC/APCI(+)-QTOFMS. Metabolomics 10:324-336.

Takahara, K., et al. (2010). Metabolome and photochemical analysis of rice plants overexpressing Arabidopsis NAD kinase gene. Plant Physiol. 152:1863-1873.

Tautenhahn, R., Cho, K., Uritboonthai, W., Zhu, Z.J., Patti, G.J., and Siuzdak, G. (2012). An accelerated workflow for untargeted metabolomics using the METLIN database. Nat. Biotechnol. 30:826-828.

Terahara, N., Saigusa, N., Ohba, R., and Ueda, S. (1994). Composition of anthocyanin pigments in aromatic red rice and its wine. J. Jpn. Soc. Food Sci. 41:519-522.

Molecular Plant 8, 58-67, January 2015 (c) The Author 2015.
Tian, S., Nakamura, K., and Kayahara, H. (2004). Analysis of phenolic compounds in white rice, brown rice, and germinated brown rice. J. Agric. Food Chem. 52:4808-4813.

Tohge, T., et al. (2011). Toward the storage metabolome: profiling the barley vacuole. Plant Physiol. 157:1469-1482.

Tong, X., et al. (2012). The rice hydroperoxide lyase OsHPL3 functions in defense responses by modulating the oxylipin pathway. Plant J. 71:763-775.

Toriyama, K., Heong, K.L., and Hardy, B. (2005). Rice is life: scientific perspectives for the 21st century. Proceedings of the World Rice Research Conference, Tsukuba, Japan. International Rice Research Institute (IRRI), Los Baños, Laguna, Philippines; Japan International Research Center for Agricultural Sciences (JIRCAS), Tsukuba, Japan. CD-ROM. 590p.

Umehara, M., Hanada, A., Magome, H., Takeda-Kamiya, N., and Yamaguchi, S. (2010). Contribution of strigolactones to the inhibition of tiller bud outgrowth under phosphate deficiency in rice. Plant Cell Physiol. 51:1118-1126.

Umehara, M., et al. (2008). Inhibition of shoot branching by new terpenoid plant hormones. Nature 455:195-200.

Vance, C.P., Uhde-Stone, C., and Allan, D.L. (2003). Phosphorus acquisition and use: critical adaptations by plants for securing a nonrenewable resource. New Phytol. 157:423-447.

Verhoeven, H.A., Jonker, H., De Vos, R.C., and Hall, R.D. (2012). Solid phase micro-extraction GC-MS analysis of natural volatile components in melon and rice. Methods Mol. Biol. 860:85-99.

Wahl, V., et al. (2013). Regulation of flowering by trehalose-6-phosphate signaling in Arabidopsis thaliana. Science 339:704-707.

Wen, W., et al. (2014). Metabolome-based genome-wide association study of maize kernel leads to novel biochemical insights. Nat. Commun. 5:3438.

Widjaja, R., Craske, J.D., and Wootton, M. (1996). Changes in volatile components of paddy, brown and white fragrant rice during storage. J. Sci. Food Agric. 71:218-224.

Wishart, D.S., et al. (2009). HMDB: a knowledgebase for the human metabolome. Nucleic Acids Res. 37:D603-D610.

Wolf, S., Schmidt, S., Muller-Hannemann, M., and Neumann, S. (2010). In silico fragmentation for computer assisted identification of metabolite mass spectra. BMC Bioinformatics 11:148.

Xie, X., et al. (2013). Confirming stereochemical structures of strigolactones produced by rice and tobacco. Mol. Plant 6:153-163.

Xiong, Y., McCormack, M., Li, L., Hall, Q., Xiang, C., and Sheen, J. (2013). Glucose-TOR signalling reprograms the transcriptome and activates meristems. Nature 496:181-186.

Xu, Z., Hua, N., and Godber, J.S. (2001). Antioxidant activity of tocopherols, tocotrienols, and gamma-oryzanol components from rice bran against cholesterol oxidation accelerated by 2,2'-azobis(2methylpropionamidine) dihydrochloride. J. Agric. Food Chem. 49:2077-2081.

Xu, Z.M., and Godber, J.S. (1999). Purification and identification of components of gamma-Oryzanol in rice bran oil. J. Agric. Food Chem. 47:2724-2728.

Yamamuro, C., et al. (2000). Loss of function of a rice brassinosteroid insensitive1 homolog prevents internode elongation and bending of the lamina joint. Plant Cell 12:1591-1606.

Yang, Z., et al. (2013). Toward better annotation in plant metabolomics: isolation and structure elucidation of 36 specialized metabolites from Oryza sativa (rice) by using MS/MS and NMR analyses. Metabolomics 10:543-555. 
Yang, Z., et al. (2014). Toward better annotation in plant metabolomics: isolation and structure elucidation of 36 specialized metabolites from (rice) by using MS/MS and NMR analyses. Metabolomics 10:543-555.

Yao, Y., Sang, W., Zhou, M., and Ren, G. (2009). Antioxidant and $\alpha$-glucosidase inhibitory activity of colored grains in China. J. Agric. Food Chem. 58:770-774.

Yawadio, R., Tanimori, S., and Morita, N. (2007). Identification of phenolic compounds isolated from pigmented rices and their aldose reductase inhibitory activities. Food Chem. 101:1616-1625.

Ye, X.D., et al. (2000). Engineering the provitamin A (beta-carotene) biosynthetic pathway into (carotenoid-free) rice endosperm. Science 287:303-305

Ying, J.Z., Shan, J.X., Gao, J.P., Zhu, M.Z., Shi, M., and Lin, H.X. (2012). Identification of quantitative trait Loci for lipid metabolism in rice seeds. Mol. Plant 5:865-875.

Yu, S.G., Nehus, Z.T., Badger, T.M., and Fang, N.B. (2007). Quantification of vitamin E and gamma-oryzanol components in rice germ and bran. J. Agric. Food Chem. 55:7308-7313.
Zeng, M.M., et al. (2012). Determination of flavor components of rice bran by GC-MS and chemometrics. Anal. Methods 4:539-545.

Zhao, X.Q., et al. (2013). Temporal profiling of primary metabolites under chilling stress and its association with seedling chilling tolerance of rice (Oryza sativa L.). Rice (N. Y.) 6:23.

Zhou, J.M., and Ibrahim, R. (2010). Tricin-a potential multifunctional nutraceutical. Phytochem. Rev. 9:413-424.

Zhou, R., Tseng, C.L., Huan, T., and Li, L. (2014). IsoMS: automated processing of LC-MS data generated by a chemical isotope labeling metabolomics platform. Anal. Chem. 86:4675-4679.

Zuther, E., Koehl, K., and Kopka, J. (2007). Comparative metabolome analysis of the salt response in breeding cultivars of rice. In Advances in Molecular Breeding Toward Drought and Salt Tolerant Crops, M. Jenks, P. Hasegawa, and S.M. Jain, eds. (Netherlands: Springer), pp. 285-315. 